\title{
Revisión preliminar del estado actual de la traducción e interpretación oficial en Colombia*
}

JUAN FELIPE ZULUAGA MOLINA**

Recepción: 10 de febrero de 2020

Aprobación: 7 de junio de 2020

Forma de citar este artículo: Zuluaga, F. (2020). Revisión preliminar del estado actual de la traducción e interpretación oficial en Colombia. Cuadernos de Lingüística Hispánica, (36), 79-102.

(doi $10.19053 / 0121053$ X.n36.2020.10814

* El presente artículo de revisión surge del proyecto de investigación "A Neo-institutionalist Study from the Sociology of the Professions to the Current Situation of Sworn Translators and Interpreters in Colombia", financiado por el CODI (Comité para el Desarrollo de la Investigación) de la Universidad de Antioquia, según el Acta n. ${ }^{\circ}$ 2019-25530 del 14 de noviembre de 2019.

** Magíster en Traducción de la Universidad de Antioquia, Escuela de Idiomas, Universidad de Antioquia, Grupo de Investigación en Traducción y Nuevas Tecnologías. Correo electrónico: juanf.zuluaga@udea.edu.co Đittps://orcid.org/0000-0001-87514992 


\title{
Resumen
}

El objetivo del presente artículo es presentar un breve estado de la cuestión sobre la producción bibliográfica y la situación actual de la traducción e interpretación oficial en Colombia. Para tal fin, se hizo una recolección y análisis de los principales referentes teóricos, normativos y prácticos relacionados con los aspectos académicos y de mercado de la actividad del traductor e intérprete oficial en Colombia y se los comparó con los que existen actualmente en el mundo. Luego de este análisis, que es apenas un planteamiento preliminar de la necesidad de un desarrollo conceptual, teórico y metodológico sobre la profesión, se reconoció la importancia de pensar en algunas iniciativas teóricas y comerciales que abran el camino a la profesionalización de esta área en el país. Asimismo, en la búsqueda se encontró que la producción académica en esta área en el país es aún muy incipiente y, en su gran mayoría, se ha centrado en temáticas relacionadas con estatus, profesionalización y legislación.

Palabras clave: traducción e interpretación oficial, estatus del traductor, traducción jurada, traducción juramentada, traducción certificada, traductor en Colombia.

\section{Preliminary Review of the Current Status of Official Translation and Interpretation in Colombia}

\begin{abstract}
The objective of this paper is to present a brief state of the art on bibliographic production and the current situation of official translation and interpretation in Colombia. For this purpose, main theoretical, normative and practical references related to academic and marketing aspects of the activity of the translator and interpreter were collected, analyzed and then compared to those existing in the world. After this analysis, which is only a preliminary approach to the need for a conceptual, theoretical and methodological development of the profession, the importance of thinking about some theoretical and commercial initiatives that pave the way for the professionalization of this area in the country was recognized. Likewise, it was found that academic production in this field in Colombia is very incipient and, in its great majority, it has focused on topics related to status, professionalization and legislation.
\end{abstract}

Keywords: official translation and interpretation; translator status; official translation;sworn translation;certificated translation; translators in Colombia. 


\section{Examen préliminaire de l'état actuel de la traduction et de l'interprétation officielles en Colombie}

\section{Résumé}

L'objectif de cet article est de présenter un bref état de la question concernant la production bibliographique et de la situation actuelle de la traduction et de l'interprétation officielles en Colombie. Pour ce faire, une quête de données et une analyse des principales références théoriques, réglementaires et pratiques liées aux aspects académiques et commerciaux de l'activité du traducteur et interprète officiel en Colombie, ont été menés à terme et comparées à ce qui existe actuellement dans le monde. Suite à cette analyse, qui correspond davantage à la nécessité d'un développement conceptuel, théorique et méthodologique de la profession, il a été reconnu qu'il est important de réfléchir à quelques initiatives théoriques et commerciales pour chercher à ouvrir la voie au besoin de lui accorder un statut conforme et réglementaire défini. Il a été constaté également qu'à partir de la recherche, la production universitaire dans ce domaine dans le pays est encore très limitée, voire pauvre, et, dans sa grande majorité, elle s'est occupée des aspects liés au statut, à la professionnalisation et à la législation.

Mots clés: traduction et interprétation officielles; statut de traducteur; traduction assermentée; traduction assermentée; traduction certifiée; traducteur en Colombie.

\section{Revisão preliminar do estado atual da tradução e interpretação oficial na Colômbia}

\section{Resumo}

0 objetivo deste artigo é apresentar um breve estado da questão referente à produção bibliográfica e à situação atual da tradução e interpretação oficial na Colômbia. Para tanto, foi realizada uma coleta e análise das principais referências teóricas, regulamentares e práticas relacionadas aos aspectos acadêmicos e de mercado da atividade de tradutor e intérprete oficial na Colômbia e comparadas com o que existe atualmente no mundo. Após essa análise, que é mais um bocado para a necessidade de um desenvolvimento conceitual, teórico e metodológico da profissão, foi reconhecido que é importante pensar em algumas iniciativas teóricas e comerciais para buscar abrir o caminho a respeito da necessidade profissionalização dessa área no país. Da mesma forma, constatou-se que, a partir da busca, a produção acadêmica nessa área no país ainda é muito incipiente e, em sua grande maioria, concentrou-se em temas relacionados a status, profissionalização e legislação.

Palavras-chave: tradução e interpretação oficial; status do tradutor; tradução juramentada; tradução juramentada; tradução certificada; tradutor na Colômbia. 


\section{Introducción}

Los traductores e intérpretes oficiales (TIO) son un grupo de profesionales, también conocidos - y a veces confundidos-como traductores legales, intérpretes de corte o jurídicos, debido a los escenarios de actuación específicos, en los que, en su mayoría, se desarrollan situaciones gubernamentales, institucionales, académicas o legales (Pym, Grin, Sfreddo \& Chan, 2012, p. 26). En general, podría decirse que son un grupo reconocido globalmente (Mayoral, 2000; Monzó, 2002; Pym et al., 2012), lo que significa que muchas instituciones e individuos saben que existen, algunas leyes los reconocen y, en algunos casos, son regulados por asociaciones, gobiernos o colegiados universitarios (Stejskal, 2003, 2005). Este reconocimiento o autorización los hace cruciales en muchas interacciones bilingües o multilingües entre instituciones y gobiernos alrededor del mundo. Además, los pone en una posición esencial para la mayoría de los contactos entre dos culturas y lenguas en algunos tipos específicos de documentos y de escenarios (Mayoral, 2000)we wish to examine official translation (termed sworn translation in Spain. Adicionalmente, los diferencia de otras modalidades en traducción y los ubica en un nivel diferente cuando se los compara con las demás modalidades que existen en esta área del conocimiento (Monzó, 2002).

En vista del reconocimiento antes expuesto, varios académicos, así como asociaciones y gobiernos en muchos países, han llevado a cabo diferentes estudios e iniciativas sobre ellos, que han descrito su comportamiento, funciones y escenarios de actuación y, en muchos casos, han intentado entender su influencia y estatus (Mayoral, 2000; Monzó, 2002; Stejskal, 2003, 2005). Adicionalmente, han aclarado la importancia de este grupo en la sociedad (De las Heras, 2017; Galanes, 2010; Vigier, Klein, \& Krestinger, 2013)y han ofrecido mejores alternativas para su formación, asociación y regulación (Ordóñez, 2009; Ortega, 2011; Zamora, 2005).

Del mismo modo, se han explorado los procesos de certificación (Galanes, 2010; García, 2007; Ortega, 2011; Vigier, 2010)y algunos aspectos históricos relacionados con esta actividad (Feria, 2007; Peñarroja, 1989a, 1989b; Peñarroja \& Cardona, 1993). Particularmente, en el caso de España, se ha examinado el estatus de los traductores jurados desde puntos de vista sociológicos, orientados al mercado, económicos, entre otros (Biguri, 2007; De las Heras, 2017; El Ghazouani, 2008; Gil \& García, 2015; Lobato, 2007; Mayoral, 1999, 2000; Monzó, 2002; Perdu \& Ridao, 2014; Salvador, 1996). De hecho, ya en otros lugares se ha explorado el concepto de estatus para estos traductores en diferentes países como en Brasil con Livio (2000), en Argentina con Cortés (2000) y Martínez y Guilman (2005), en Costa Rica con Zamora (2005), en Rusia con Guzmán 
(2008), en Canadá con Hlavac (2013) y en España y Francia con Barceló y Delgado (2016), entre otros.

Al contrastar todas estas iniciativas y estudios en el mundo relacionados con los traductores e intérpretes oficiales (en adelante TIO), se puede encontrar que en Colombia solamente hay seis reflexiones académicas que hablan específicamente de este segmento de traductores/intérpretes (Clavijo, 2011; Martín, 2013; Quiroz, Gómez \& Zuluaga, 2013; Quiroz, Zuluaga, \& Gómez, 2015; Quiroz \& Zuluaga, 2014; Zuluaga \& Quiroz, 2018). Así como los estudios realizados en otros países diferentes a España, las reflexiones son sobre todo de corte demográfico o descriptivo. Quiroz et al. (2013), por ejemplo, en un estudio de corte cuantitativo, se enfocaron en la definición del perfil de los candidatos a TIO, mientras que Quiroz et al. (2015) se enfocaron en aspectos demográficos de los TIO a partir de una encuesta y de una reflexión sobre la legislación existente sobre este grupo. Zuluaga y Quiroz (2018) hicieron una descripción cuantitativa del desempeño de los candidatos que querían hacerse TIO, y Clavijo (2011) y Martín (2013), por su parte, hicieron una descripción acerca de problemáticas relacionadas con aspectos prácticos y legislativos en la traducción jurídica y, por ende, oficial. Sin embargo, tal como puede verse, los estudios e iniciativas en este segmento de traductores en Colombia son todavía incipientes y podría decirse que es un área del conocimiento que se encuentra aún en florecimiento y construcción.

Una reflexión sobre este segmento de traductores 0 , al menos, un acercamiento a su estado actual en términos académicos, pero también en términos prácticos, es decir, en lo que respecta al estado de la actividad profesional como tal, podría dar algunas luces sobre las problemáticas que tiene este conglomerado y, al mismo tiempo, podría permitir a la academia determinar algunas de las principales necesidades y claridades sobre el camino que se debe recorrer tanto en el ámbito público como privado en lo que respecta a su desarrollo profesional.

Por todo lo anterior, el presente artículo hace un recorrido bibliográfico y práctico por las diferentes realidades actuales de la profesión de TIO en Colombia y, para tal fin, presenta un estado de la cuestión con diferentes elementos bibliográficos y de conocimiento público que permiten acercarse al fenómeno de la traducción e interpretación oficial en Colombia y contextualizarlo en el campo de la traducción general. Para la construcción del presente capítulo se revisaron alrededor de 200 referentes teóricos y normativos y, a partir de su análisis y categorización, se presentan los más relevantes en tres apartados: aspectos académicos y de producción bibliográfica, aspectos normativos y legales y procesos de certificación (exámenes para traductor e intérprete oficial). Cada apartado 
del presente artículo tendrá algunos comentarios propios del proceso de lectura y de análisis y, de forma adicional, una pequeña conclusión sobre lo encontrado.

\section{La traducción e interpretación "oficial” en Colombia}

Así como en otros contextos, la traducción e interpretación oficial en Colombia es muy diversa. De hecho, esa designación de "oficial", que es la que se encuentra comúnmente en la legislación, puede comprender tres grandes grupos de traductores o intérpretes en un sentido amplio. El primer grupo es el de traductores e intérpretes oficiales desde y hacia lenguas orales que no son oficiales ni cooficiales en el territorio, como francés, italiano, inglés, entre otras; un grupo que ingresa a la actividad profesional exclusivamente a través de un examen de aprobación de sus conocimientos en traducción e interpretación en ambas vías -lengua adicional hacia español y español hacia lengua adicional- el cual es regulado por los decretos 382 y 2257 (Diario Oficial de Colombia, 1951b; Diario Oficial de Colombia, 1951a). Tal como se ha mencionado, este grupo es, de forma común, denominado "de traductores jurados, públicos, juramentados", entre otros, con el fin de eliminar cualquier ambigüedad sobre la tarea específica dentro del área de la traducción/interpretación que estos desarrollan.

El segundo grupo son los intérpretes desde y hacia lengua de señas colombianaespañol, quienes tienen una regulación específica por medio del Decreto 2369 (Diario Oficial de Colombia, 1997) y la Resolución 05274 (Diario Oficial de Colombia, 2017). Un grupo que hasta hace poco no tenía ninguna regulación específica sobre aspectos tan importantes como las exigencias académicas para el acceso a la actividad remunerada y que ahora, gracias a esta nueva resolución, lo hacen por medio de un proceso de certificación -proceso ya existente- o por medio de la certificación de un proceso de formación avalado por el Ministerio de Educación.

Un tercer y último grupo es el de traductores o intérpretes desde y hacia español- lenguas nativas o ancestrales, que son cooficiales con el español en el territorio colombiano. Dicho grupo cuenta también con una regulación específica estipulada en la Ley 1381 (Diario Oficial de Colombia, 2010). A pesar de esta regulación, hay diferentes matices importantes que habría que evaluar al explorar académicamente este grupo, debido a que, la mayoría de las veces, la "autorización" para que sean traductores 0 intérpretes se da por un criterio de aceptación de las mismas comunidades de las que hacen parte y no por medio de una formación académica -como es el caso del segundo grupo-, o por un proceso de certificación -como es el caso del primer grupo-. Ahora bien, aunque evidentemente este no es el foco de la presente investigación, sería muy relevante indagar sobre este grupo de profesionales en Colombia, puesto que, solo en términos de 
organización como actividad remunerada 0 , al menos, autorizada en el territorio, es una de las más apoyadas y estabilizadas en el contexto jurídico, sociocultural y de oficialidad ${ }^{1}$.

Para los objetivos y líneas específicas de concentración y análisis del presente artículo, será el primer grupo -traductores e intérpretes oficiales de lenguas orales que no son oficiales ni cooficiales en el país- el que será analizado y problematizado. Para este grupo en particular son los decretos 382, 722 y 2257 (Diario Oficial de Colombia, 1951b; Diario Oficial de Colombia, 1951a; Diario Oficial de Colombia, 1982), así como la Ley 962 (Diario Oficial de Colombia, 2005) (Quiroz et al., 2013) los que nos indican que, como tal, son un grupo o conglomerado al cual debe llamarse o designarse "oficial".

Desde hace décadas, el Ministerio de Relaciones Exteriores se ha dado a la tarea de mantener una lista de los traductores e intérpretes oficiales calificados en el territorio. Dicha lista se podía encontrar hasta hace no más de un año (2020) en el propio sitio web de la Cancillería (s.f.); no obstante, debido a diferentes circunstancias y cambios que acaecieron en el transcurso de los últimos años y que deberían ser objeto de discusión en una investigación posterior, dicha lista desapareció y es ahora imposible, desde un punto de vista formal o institucional, definir cuál es la cantidad exacta 0 quiénes son puntualmente los traductores e intérpretes oficiales colombianos. A pesar de esta realidad, según estimaciones de tres investigaciones publicadas en la última década en las que se indica la cantidad de TIO que estaban inscritos en esta plataforma al momento de su consulta (en casi todos los casos las consultas se realizaron después del 2010) (Clavijo, 2011; Quiroz et al., 2013, 2015), se observaba que había entre 700 y 900 registros de personas con esta certificación.

Adicional a este elemento cuantitativo, en un estudio realizado por Quiroz et al. (2013) se especifican algunos elementos demográficos a partir del análisis de dicha plataforma o directorio, de la legislación y de una encuesta a 38 traductores e intérpretes oficiales en todo el país. Dichos datos son de utilidad como punto de partida de la presente reflexión y podrían resumirse, para lo que interesa en este segmento -aspectos demográficos y sociales- de la siguiente manera:

- La ubicación geográfica de los TIO encuestados es mayoritariamente en Cundinamarca, un elemento que se confirma con el contraste con 610 registros de TIO del Directorio del Ministerio de Relaciones Exteriores, en el que su gran mayoría se encuentra en este departamento (generalmente Bogotá);

1 Véase, por ejemplo, la traducción a 47 lenguas nativas del Acuerdo de Paz con las FARC (Alto Comisionado para la Paz, 2018). 
- hay predominancia en la actividad remunerada hacia el sexo femenino entre los TIO, aunque no de una forma desequilibrada con respecto a la cantidad de hombres;

- la tendencia es que los TIO sean de una edad superior a los 45 años;

- en un porcentaje alto, sus estudios son bastante diversos, aunque muchos de ellos se inclinan sobre todo por las lenguas, las artes y las humanidades;

- en su mayoría trabajan como independientes y hay una tendencia evidente a tener una dedicación horaria superior a las 20 horas semanales a tareas relacionadas con la traducción e interpretación oficial;

- los TIO se desempeñan en actividades de traducción o interpretación oficial y en actividades como docencia, actividades relacionadas con el sector comercial 0 financiero 0 actividades relacionadas con el sector jurídico;

- en promedio, las lenguas de trabajo y conocimiento son el inglés, el francés, el italiano y, en menor medida, el alemán y otras lenguas europeas o asiáticas. Asimismo, se observa que hay traductores que expresan conocimiento o trabajo en combinaciones con más de una lengua adicional al español;

- los TIO no se encuentran, en su mayoría, asociados a ninguna entidad gremial;

- el "voz a voz" es su garantía en el flujo de trabajo, no hay mucha actividad o interés en el desarrollo de estrategias de promoción de los servicios.

El anterior resumen surge de la revisión de los datos del artículo y de las conclusiones y presentación de los resultados que los autores hacen dentro del mismo. En efecto, la impresión que queda de las constataciones encontradas por los autores es apoyada por fuertes conclusiones en torno al hecho de que la traducción e interpretación oficial se mantienen, así como la traducción e interpretación no oficial, como actividades remuneradas liberales (Quiroz et al., 2013).

\section{Aspectos académicos}

En un estudio reciente sobre los diferentes programas de formación vigentes 0 no vigentes en Colombia, los docentes Gómez y Gómez (2011, p. 140) hacen un recorrido por más de siete universidades en las que se ofertan formaciones en traducción o interpretación. En dicho estudio, así como en otros (Gómez, 2019), que sirven de referencia en este artículo para observar, en general, cuál es la oferta de formación en 
el país, se aprecia que, de las diez posibilidades de formación, apenas cinco de ellas se encuentran aún vigentes. Esta realidad se contrasta con el hecho de que, actualmente, esta oferta no ha variado mucho: solamente una universidad ofrece un pregrado Universidad de Antioquia- y cuatro universidades tienen activas las líneas de formación posgradual - Universidad de Antioquia, Universidad Autónoma de Manizales, Universidad del Rosario y Universidad de Pamplona. Tal como se indica en ese estudio, la presencia de un componente en traducción científica y técnica no es una constante visible en los programas de formación.

La falta de oferta en el componente científico-técnico no es exclusiva de esta especialidad. Cuando se observó en detalle la oferta de estos programas, tampoco se encontró ninguna formación específica en traducción o interpretación oficial, ni siquiera dentro de los microcurrículos de los cursos de traducción legal o comercial de algunos de los planes de estudio, excepto para el curso de "Textos jurídicos y comerciales" ofrecido en la Universidad de Antioquia.

A este vacío curricular en pregrado y posgrado se suma el hecho de que solo se encontró, en lo que tiene que ver con educación no formal o educación para el trabajo, un curso de preparación para el examen para traductor e intérprete oficial, el cual es ofrecido por la Pontificia Universidad Javeriana, en el que, entre otros, se tienen en cuenta aspectos como:

- definición y alcances de la traducción oficial;

- estructura, procedimientos y criterios del examen de Traducción e Interpretación Oficial; $y$,

- habilidades de traducción y conocimientos lingüísticos, terminológicos y jurídicos que debe poseer un candidato al Examen (Universidad Javeriana, s.f.).

En resumen, en lo que tiene que ver con oferta académica para traducción e interpretación oficial no existe en el escenario universitario ni gremial un proceso institucional con respaldo para los que se dedican o buscan dedicarse a esta actividad.

Lo anterior se evidencia o se acentúa en el contexto de la producción bibliográfica relacionada con esta área y los grupos de investigación o individuos dedicados a la misma en el país. Aunque la revisión fue exhaustiva, se aclara que pudieron haber quedado algunas referencias sin ser exploradas, puede observarse que hay alrededor de 16 
publicaciones relacionadas con la traducción/interpretación oficial en el país, distribuidas de la siguiente manera:

- Grupo de investigación en traducción y nuevas tecnologías: en su línea de aspectos sociales y profesionales de la traducción se pueden encontrar los siguientes productos bibliográficos en los que se aborda el tema de la traducción e interpretación:

- Libro: Estatus actual del traductor en Colombia, publicado por Gabriel Quiroz, Juan Felipe Zuluaga y Norman Gómez (2015). Esta obra presenta un capítulo entero sobre la traducción e interpretación oficial en Colombia. Hace énfasis en el estatus social y legal del traductor y, metodológicamente, se sirve de elementos de un enfoque cuantitativo (estadística descriptiva) para presentar el estado de estos profesionales.

- Artículo: "Candidatos a traductor e intérprete oficial en Colombia: profesionales u oportunistas", publicado en la revista Babel por Gabriel Quiroz y Juan Felipe Zuluaga (2014). Este artículo reflexiona por medio de análisis cuantitativos sobre el desempeño y el perfil de los candidatos a TIO que han participado en la convocatoria hecha por la Universidad de Antioquia.

- Artículo: "Panorama general del traductor e intérprete oficial en Colombia", publicado en la revista Núcleo por Gabriel Quiroz, Juan Felipe Zuluaga y Norman Gómez (2013). Contiene, como se ha indicado, un bosquejo del estado actual de la actividad remunerada, basado en una encuesta y en la recolección de datos del Directorio de TIO del Ministerio de Relaciones Exteriores y de la legislación sobre este tema en Colombia.

- Artículo: "Análisis del desempeño de candidatos a traductor e intérprete oficial en Colombia", publicado en la revista Cadernos de Tradução por Juan Felipe Zuluaga y Gabriel Quiroz (2018). En este artículo se analizan los datos de los resultados de los candidatos a traductor e intérprete oficial de la Universidad de Antioquia. En él se perfilan algunos elementos relacionados con el desempeño contrastado con elementos demográficos y se reflexiona 
sobre el estado de la cuestión en lo que tiene que ver con competencias y necesidad de la traducción e interpretación oficial en Colombia.

- Artículo: "Hacia un perfil profesional del traductor en Colombia", publicado en la revista de la Escuela de Administración y Negocios de la Universidad EAN y escrito por Liliana Franco y Gabriel Quiroz (2011), explora perfiles de los traductores e intérpretes en Colombia y comenta algunos detalles sobre los TIO y la traducción e interpretación oficial en Colombia; en ambos casos se hacen acotaciones demográficas y del comportamiento del mercado.

- Otras publicaciones: "Certificarse como traductor e intérprete oficial" (Zuluaga, 2014) y "Requisito: traducción oficial" (Zuluaga, 2015) y "Traducción e interpretación oficial, en el limbo" (Echeverri \& Zuluaga, 2019) publicadas en el periódico Alma Máter de la Universidad de Antioquia o en su página web, y "Traducción e interpretación oficial en Colombia" (Zuluaga, 2019) publicado en el Periódico Digital de la Universidad Nacional de Colombia, cuyos temas hacen alusión a los procesos de certificación actuales y dan pistas sobre el estado actual del servicio de la traducción/ interpretación oficial en Colombia.

- Tesis de maestría sobre la traducción o interpretación oficial: para esta revisión se exploraron los repositorios de cuatro universidades: la Universidad de Antioquia, la Universidad EAN, la Universidad Autónoma de Manizales y la Universidad de Pamplona. Se encontró lo siguiente:

- Ni en la Universidad de Antioquia ni en la Universidad Autónoma de Manizales se encontró una tesis o reflexión posgradual relacionada con la traducción e interpretación oficial.

- En la Universidad de Pamplona se encontró una tesis de posgrado en la que se reflexiona acerca del estatus de intérprete en Colombia: "Analysis of the Current Status of Interpreting as a Profession in Colombia: An Exploratory Case Study", tesis de maestría desarrollada por la estudiante María Fernanda Padilla (Padilla, 2018) en la que se aborda, por medio de una encuesta, el estatus actual de los intérpretes en Colombia. 
- En la Universidad EAN se hallaron diferentes publicaciones que trataban de forma tangencial la traducción e interpretación oficial:

- "Análisis del proceso metodológico de la traducción jurídica caso Editorial Temis S.A." publicada en (2012) y desarrollada en el marco de una formación en Traducción por Andrea Deantonio Motta. Este trabajo de grado explora elementos de corte jurídico, específicamente, y hace mención de la traducción e interpretación oficial en el contexto y relevancia de la traducción jurídica.

- "Estado actual del mercado de la interpretación en Bogotá" (2013), este trabajo de grado se aproxima desde un enfoque cuantitativo al estado de la interpretación oficial en Bogotá. Para tal fin, Irma Alexandra Montealegre hace una encuesta a 20 intérpretes para determinar aspectos básicos demográficos y, sobre todo, profesionales (proceso de interpretación, herramientas utilizadas, entre otros).

- "Traducciones jurídicas: competencias del traductor e impacto en su interpretación” (2013). Este trabajo de grado, realizado por Johann David Martín Bermúdez, busca describir algunas de las competencias de los traductores jurídicos y su impacto en el proceso de interpretación, traducción de los textos.

- "El papel de los traductores en los tratados de libre comercio (Colombia-Estados Unidos)", publicado por Infante y Mora (2012), se trata de un trabajo de grado realizado con el fin de evaluar el papel de los traductores en los tratados de libre comercio entre Colombia y Estados Unidos. Aunque no se menciona específicamente, es evidente la presencia de traductores e intérpretes oficiales en este estudio y es por esto que se agrega a la presente colección de referencias en Colombia para la traducción e interpretación oficial.

- "Perfil del traductor: incidencia de su formación en el desempeño profesional" (2013). Este trabajo de grado de Lorena Ortiz Rodríguez y Ana Sanabria Díaz explora el impacto de los procesos de certificación y 
de la formación de los traductores en su desempeño profesional. En lo que respecta a la traducción e interpretación oficial, reflexiona sobre los procesos de certificación y comenta generalidades acerca de la actividad en cuestión.

- Otras publicaciones en revistas académicas o profesionales relacionadas con traducción e interpretación oficial:

- Publicaciones en las que se mencionan aspectos de la traducción e interpretación oficial en Colombia:

- Babel en las empresas colombianas: una mirada actual de la traducción, (2008), en este libro se hace una reflexión sobre "el perfil y la oferta en el mercado para los traductores en Colombia" (Cerón, 2009, p. 153). Los autores (Clavijo, Mendoza, Franco, Duque \& Rodríguez, 2008) reflexionan también sobre la traducción e interpretación oficial en su libro, sobre todo en lo que tiene que ver con los aspectos profesionales y de mercado.

- "Necesidad de la traducción en las empresas: procesos y perfiles", publicado en la revista de la Escuela de Administración y Negocios de la Universidad EAN (2006). Este artículo, de los profesores Bibiana Clavijo, María Claudia Panqueva, María Clara Correal, María Isabel Duque, Edgar Mendoza, Carlos Franco y Jairo Alberto Galindo, explora la legislación y la existencia de la traducción e interpretación en Colombia y examina, en términos generales, aspectos de mercado de esta actividad.

- "Algunos apartes de la traducción jurídica y oficial en Colombia", publicado en la revista de la Escuela de Administración y Negocios de la Universidad EAN (2011), en este artículo la profesora Clavijo reflexiona sobre aspectos sociales, gremiales y políticos de los traductores e intérpretes oficiales y jurídicos en Colombia y concluye que es importante repensar los procesos de formación y certificación en esta especialidad.

- "iHacia el camino de la profesionalización de la traducción gracias a la futura NTC?" publicado en la revista Mutatis Mutandis (2009). Esta reflexión de 
Jeannette Insignares hace alusión a la traducción e interpretación oficial y al eventual impacto de la norma NTC sobre traducción e interpretación que surgió en esa época en el ejercicio de la actividad. La mención a la traducción e interpretación oficial en este contexto es enunciativa.

Luego de esta breve recapitulación sobre la producción bibliográfica en traducción e interpretación oficial en Colombia, es importante resaltar algunas conclusiones importantes:

- A pesar de que las publicaciones acerca de esta área 0 especialidad de la traducción en Colombia son recientes -casi todas fueron hechas en los últimos diez a quince años-, hay un interés evidente por reflexionar y hacer un llamado a visibilizar esta actividad por medio de la investigación.

- Hay tres vertientes evidentes en los estudios llevados a cabo en esta especialidad en Colombia: a. aspectos demográficos y de estatus del profesional -reflexiones y análisis de investigación cuantitativa-; $b$. aspectos de comportamiento del mercado y de empresa; y c. aspectos relacionados con el candidato a TIO.

- Hay una tendencia generalizada a utilizar enfoques netamente cuantitativos en la investigación de este grupo de profesionales.

- Las reflexiones sobre los aspectos normativos son frecuentes y, en general, se han centrado en la presentación de las legislaciones actuales y en explicar de qué manera estas tienen un impacto o influencia en la percepción del TIO desde la legislación.

\section{Procesos de certificación}

En sintonía con los procesos de certificación en el mundo, los procesos de certificación en Colombia están revestidos de una particularidad local según lo revisado para la bibliografía de esta área. En este caso, las particularidades, que no son comunes a otros procesos de certificación, se podrían resumir, y aplica para todos los candidatos a esta certificación (Zuluaga \& Quiroz, 2018), de la siguiente manera:

a). evaluación de la competencia oral [interpretación] y la competencia escrita [traducción];

b). evaluación en ambas direcciones: español hacia lengua adicional y viceversa; 
c). presencia de textos especializados y del discurso general;

d). exposición al candidato a escenarios de traducción/interpretación reales a los que eventualmente se puede enfrentar;

e). ausencia de requisitos de cualquier índole para acceso al examen.

Con el fin de ampliar la información de los cinco puntos anteriormente enunciados, en la presente subsección se explicará de forma sucinta la estructura de los exámenes ofrecidos en las entidades avaladas para llevarlos a cabo.

En primer lugar, tal como se puede observar en las páginas web de cada una de las entidades (Universidad de Antioquia, en Medellín, y Universidad Nacional de Colombia, en Bogotá), los exámenes evalúan competencias orales y competencias escritas. En el caso de la Universidad de Antioquia, la competencia oral se evalúa por medio de la exposición al candidato a un escenario de interpretación a vista y a una interpretación alterna; y en el caso de la Universidad Nacional, la exposición se da en el escenario de una interpretación alterna únicamente. La evaluación de la competencia escrita, en ambas universidades, se enfoca, en primer término, en el amplio conocimiento de la traducción de textos especializados en el área jurídica y, en segundo término, en el área científica 0 técnica -algo exclusivo de la oferta del examen de la Universidad de Antioquia. Para ambos exámenes, el proceso de revisión se lleva a cabo bajo criterios básicos de corrección de traducciones/interpretaciones (para más detalles sobre los exámenes, ver Zuluaga \& Quiroz, 2018) y con taxonomías creadas por las comisiones de cada una de las entidades.

Un elemento jurídico interesante que contrasta 0 supone una discusión adicional sobre lo que se acaba de exponer, es lo que aparece en el Decreto 722 de 1982, artículo 2, el cual define aspectos temáticos acerca de la composición del examen que imparten las instituciones avaladas. En el decreto se establece que:

Los exámenes para los aspirantes a intérpretes oficiales versarán sobre dos evaluaciones, una oral y otra escrita. En la oral se tendrán presentes los siguientes aspectos: a) Habilidad del candidato para leer en la lengua extranjera con corrección en la pronunciación y en la entonación. b) Habilidad para traducción consecutiva de la lengua extranjera al español y viceversa, con exactitud en las ideas y fluidez en el campo del idioma. c) Habilidad para captar el sentido general y retener las ideas principales de un texto escuchado. En la evaluación escrita se tendrán en cuenta los siguientes aspectos: a) Habilidad del candidato para reconocer cognados falsos. b) Habilidad para traducir del idioma extranjero al 
español y viceversa con exactitud en el sentido, corrección ortográfica y naturalidad en el manejo de los dos idiomas. c) Habilidad en el uso del diccionario.

En lo que tiene que ver con la dirección, la certificación en Colombia exige que el candidato demuestre su capacidad para traducir e interpretar hacia y desde su lengua adicional de elección y el español. Este elemento, bastante particular cuando se compara con la realidad de otros contextos, es interesante desde la teoría, en primer lugar, por la tradicional creencia de que los traductores deberían traducir hacia su lengua materna preferible y exclusivamente (Pavlović, 2013), y, en segundo lugar, en lo que respecta al comportamiento del mercado. En un apartado más adelante se discutirá a fondo este concepto y se intentará dar algunas luces acerca de su comportamiento en el escenario o el mercado de la traducción e interpretación oficial.

Un tercer elemento que es fundamental poner sobre la mesa de discusión es la presencia de textos orales y escritos de índole especializada y del discurso general. Es fundamental por dos razones: ante todo, el que se evalúe con este tipo de textos indica -o supone-, grosso modo, que el comportamiento del mercado de la traducción e interpretación oficial se mueve en estos discursos. La discusión adicional sobre el hecho de que sean estos los tipos de textos (jurídico-comerciales y de ciencias exactas, naturales, en su mayoría) es que ha sido así desde su creación y, según información de las comisiones, no ha cambiado mucho durante los años ni está en proceso de evaluación para que cambie. Todo esto, a pesar del cambiante mercado de la traducción en Colombia y en el mundo (Zuluaga \& Quiroz, 2018).

La traducción de estos textos, así como la interpretación que se lleva a cabo en el escenario de los exámenes, buscan ser la representación de situaciones reales a las que eventualmente un traductor e intérprete oficial en ejercicio se podría enfrentar. Es común, por ejemplo, ver que en los exámenes se incluyan registros civiles de nacimiento, diplomas o demandas, documentos bastante frecuentes en el trabajo cotidiano de los traductores oficiales, entre otros (Zuluaga \& Quiroz, 2018).

Por último, y como una discusión/reflexión final que ya ha sido objeto de discernimiento en otros espacios, es importante mencionar que, debido a que no está presente en la ley y a que ninguna de las comisiones o agremiaciones ha levantado la voz al respecto, cualquier persona sin importar si es nacional o extranjera, profesional o bachiller, o cualquier otro elemento de perfil, puede presentarse al examen. En un artículo de Zuluaga y Quiroz (2018), se reflexiona sobre este aspecto y se dejan entrever algunas de las problemáticas ligadas a esta libertad en el acceso a la profesión, sobre todo 
en lo que tiene que ver con los entes certificadores y la logística para el desarrollo del examen.

\section{Aspectos normativos}

Hasta la finalización del presente artículo no se encontró ninguna ley que le dé un reconocimiento profesional más allá de la figura legal para asuntos diplomáticos y judiciales al traductor e intérprete oficial en Colombia. De hecho, Colombia, a diferencia de otros países latinoamericanos (Zamora, 2005), no tiene una legislación para proteger al traductor e intérprete; todo se reduce a unos pocos artículos en los que se define someramente la función de perito y traductor oficial, tal como se observa en varios documentos oficiales como el Código de Procedimiento Penal (Ley 906 de 2004): Testigo de lengua extranjera, artículo 427. Documentos procedentes del extranjero, artículo 428. Traducción de documentos; en una disposición del Congreso (Ley 962 de 2005, art. 33, el cual modifica el artículo $4 .^{\circ}$ del Decreto 382 de 1951) que habla del examen para el ejercicio del oficio de traductor e intérprete oficial en Colombia, entre otras.

A pesar de las constataciones anteriores -ausencia de ley de protección a la profesión y aparición/comprensión del traductor como perito bilingüe-, la legislación colombiana incluye una normativa extensa entre leyes, decretos y otro tipo de normas (alrededor de 160, desde 1951 hasta 2012, según un rastreo realizado por Quiroz et al., 2013) que tienen que ver de alguna manera con la traducción en general. Dicha normativa está orientada principalmente a garantizar los derechos de los ciudadanos para acceder a documentos traducidos o a intérpretes que los representen en diferentes escenarios. No obstante, en cuanto a los traductores, son pocas las leyes que se refieren a sus derechos en particular²; lo que es más evidente en el segmento de los traductores oficiales donde apenas unas cuantas leyes están relacionadas con la profesión como tal.

En términos generales, podría decirse que el panorama jurídico para los traductores oficiales es bastante variado, en primer lugar, por las diferentes formas como son nombrados y citados a lo largo de los artículos y de las normas y, en segundo lugar,

2 Según lo explorado en un capítulo del libro Estatus actual del traductor en Colombia, publicado por Quiroz et al. (2015), podría decirse que solo la Ley sobre Derechos de Autor que existe en Colombia da algunos derechos sobre el traductor de lenguas no indígenas ni de señas directamente (p. 53). No obstante, como se puede observar en la normativa recogida en ese capítulo, la forma de dar derechos -sobre todo de acceso laboral- a los traductores está dada casi siempre por una necesidad de cubrir un deber con el ciudadano por parte del Estado (Quiroz et al., 2015, p. 52). Algunos ejemplos de esto están consignados en el artículo publicado en la revista Núcleo (Quiroz et al., 2013), donde se hace alusión, p. ej., a más de 25 normas como el Decreto 2550 de 2010, el Decreto 1861 de 2006 u otros (Quiroz et al., 2013, p. 176), en las que se muestra cierta protección a los derechos de los ciudadanos en lo que respecta a la actividad que debe realizar un traductor oficial y no cualquier persona con competencia en lengua extranjera. 
por las diferentes funciones en las que son mencionados como agentes obligatorios en la resolución de situaciones específicas (aspectos jurídicos, diplomáticos, empresariales, entre otros). Según Quiroz et al. (2013) esto es algo que se debe explorar (Quiroz et al., 2013, p. 177) en términos de sus funciones y su poco reconocimiento con miras a reconocerlos como agentes únicos y necesarios en procesos en los que dos lenguas se encuentran. Un ejemplo de esta dificultad en la denominación es: si en una norma se exige presentar un documento traducido oficialmente, en el artículo se puede hacer referencia al traductor oficial en la forma de "traducción oficial", lo que refleja la función del traductor y no hace referencia directa a este como agente, a pesar de que en otras normas se dice expresamente, remítase a un traductor oficial para este encargo. Hay cierta invisibilización del sujeto por medio de la preponderancia de la descripción de su función.

En la Gráfica 2 de la investigación publicada por Quiroz et al. (2013, p. 176), se muestra que, generalmente, en las normas se hace referencia a la traducción e interpretación oficial como una profesión o un "servicio" que es necesario en muchas ocasiones en las que se presentan relaciones con países o comunidades que no son hispanohablantes y que la mención más común en las normas es "traducción oficial". En otros casos, la referencia a la presencia o acción de un traductor oficial es más difusa, como, por ejemplo, en las que aparece la designación "traducir", cuyas relaciones con la oficialidad pueden variar según el contexto, pues podrían necesitar bien sea de traductores e intérpretes oficiales como de traductores o intérpretes que no lo son. No obstante, estos escenarios tienen una constante y es que hacen referencia normalmente a situaciones en las que hay intervención gubernamental directa, es decir, del sector público.

Por último, es importante mencionar en este breve recorrido por la legislación en traducción e interpretación oficial un avance importante en lo que tiene que ver con la regulación de la actividad profesional. Para ser más precisos, se trata del único avance, aunque muy significativo. Ocurrió en 2010 y se refiere a la aprobación de la Norma Técnica Colombiana 5808: Servicios de traducción. Requisitos para la prestación del servicio, por parte del Comité Técnico 218 — Terminología y otros recursos lingüísticos y de contenido - del Instituto Colombiano de Normas Técnicas (ICONTEC, 2010). En dicha norma, que cubre diferentes aspectos de la práctica de la traducción en Colombia, se agrega un anexo sobre la traducción oficial de carácter informativo.

\section{Anotaciones finales}

En primer lugar, la enunciación de los elementos que aparecen en este artículo permitieron sentar algunas bases de la contextualización y la presentación del panorama 
general de la traducción e interpretación oficial en Colombia y en el mundo, lo que, a su vez, puede permitir tener una mayor claridad para futuras investigaciones en las que se aborde este tema y además puede servir de insumo para legislar sobre traducción e interpretación oficial en el país. También es importante mencionar que, a pesar de lo exhaustiva de esta pesquisa, es normal que haya habido algunas incorrecciones 0 que haya hecho falta nombrar algunos elementos. En ningún momento este artículo pretende ser absoluto, es decir, no busca presentar toda la información existente en el área de la traducción e interpretación oficial, sino que, por el contrario, busca exponer algunos puntos controversiales y abrir camino en lo que respecta a diferentes áreas de reflexión sobre las que se podrían generar iniciativas de investigación y profesionalización en el país y, por qué no, en algunos países de América Latina donde esta profesión aún no cuenta con una regulación ni organización como sí se puede observar en países de Europa y Norteamérica.

\section{Referencias}

Alto Comisionado para la Paz (2018). El Acuerdo de Paz se habla en lenguas nativas. Recuperado de http://www.altocomisionadoparalapaz.gov.co/herramientas/Paginas/acuerdo-lenguasnativas/El-Acuerdo-de-Paz-se-habla-en-lenguas-nativas.aspx

Barceló, M., \& Delgado, I. (2016). La traduction assermentée en Espagne et en France: Aspects privés et étude comparative. Revista de Llengua i Dret, 66, 124-135.

Biguri, K. (2007). La situación actual de la traducción jurada en lengua vasca. Papers Lextra, 3, $37-40$.

Cerón, A. (2009). Reseña: Babel en las empresas colombianas: una mirada actual de la traducción. Comunicación, Cultura y Política, 1(1), 153-154.

Clavijo, B. (2011). Algunos apartes de la traducción jurídica y oficial en Colombia. Revista EAN, $70,58-67$.

Clavijo, B., Mendoza, E., Franco, A., Duque, M., \& Rodríguez, E. (2008). Babel en las empresas colombianas: una mirada actual de la traducción. Bogotá: Universidad EAN. https:// doi.org/10.21158/01208160.n58.2006.387

Clavijo, B., Panqueva, M. C., Correal, M. C., Duque, M. I., Mendoza, E., Franco, C., \& Galindo, J. A. (2006). Necesidad de la traducción en las empresas. Revista EAN, 58, 61-78.

Cortés, G. (2000). Régimen legal de la traduccíon y del traductor público. Íkala, Revista de Lenguaje y Cultura, 5(9-10), 159-162. 
de las Heras, M. (2017). La profesión de traductor jurídico en España: Titulación y conocimientos exigidos para su ejercicio a raíz de la creación del título universitario de Licenciado en Traducción e Interpretación en 1991. FITISPos International Journal, 4, 49-68.

Deantonio, A. (2012). Análisis del proceso metodológico de la traducción de la traducción jurídica (trabajo de grado de maestría). Universidad EAN. Recuperado de https:// repository.ean.edu.co/bitstream/handle/10882/864/DeantonioAndrea2012. pdf? sequence $=1 \&$ isAllowed $=\mathrm{y}$

Diario Oficial de Colombia. (1951a). Decreto 2257. Bogotá. Recuperado de http://www.leyex.info Diario Oficial de Colombia. (1951b). Decreto 382. Bogotá. Recuperado de http://www.leyex.info Diario Oficial de Colombia. (1982). Decreto 722. Bogotá. Recuperado de http://www.leyex.info Diario Oficial de Colombia. (1997). Decreto 2369. Bogotá. Recuperado de http://www.leyex.info Diario Oficial de Colombia. (2005). Ley 962. Bogotá. Recuperado de http://www.leyex.info Diario Oficial de Colombia. (2010). Ley 1381. Bogotá. Recuperado de http://www.leyex.info

Diario Oficial de Colombia. (2017). Resolución 05274. Bogotá. Recuperado de http://www.leyex. info

Echeverri, J., \& Zuluaga, J. (2019). Traducción e intepretación en Colombia, en el limbo. UdeA Noticias virtuales. Recuperado de http://bit.ly/21SCp8U

El Ghazouani, A. (2008). El mercado profesional en traducción jurídica y jurada y en mediación intercultural: El caso de la Lengua Árabe. Tonos Digital, 15, 1-18.

Feria, M. (2007). La interpretación judicial y la traducción jurada árabe-español en Málaga durante los años noventa. Puentes, 8, 25-29.

Galanes, I. (2010). La acreditación de traductores y/o intérpretes jurados en España: Novedades, contrastes e incoherencias. Sendebar, 21, 251-270.

García, M. (2007). La habilitación de traductores jurados en Galicia. Papers Lextra, 3, 31-36.

Gil, I., \& García, S. (2015). El futuro de la traducción jurada propuesta: El colegio profesional de traductores-intérpretes jurados. Miscelánea Comillas, 73(143), 447-462.

Gómez, N. (2019). La formación de traductores en Hispanoamérica: un vistazo a los programas de grado en traducción en seis instituciones de Argentina, Colombia y Venezuela. Mutatis Mutandis, 12(1), 11-50. https://doi.org/10.17533/udea.mut.v12n1a01 
Gómez, N., \& Gómez, J. I. (2011). Aproximación a una didáctica de la traducción de textos científicos y técnicos inglés-español. Íkala, Revista de Lenguaje y Cultura, 16(27), 135163.

Guzmán, R. (2008). La traducción jurada en Rusia: situación actual y perspectivas. Sendebar, 19, 189-198.

Hlavac, J. (2013). A cross-national overview of translator and interpreter certification procedures. Translation and Interpreting, 5(1), 32-65. https://doi.org/10.12807/ti.105201.2013.a02

ICONTEC. NTC 5808 (2010). Bogotá: ICONTEC. Recuperado de https://www.mineducacion.gov. c0/1621/articles-157089_archivo_pdf_NTC_5580.pdf

Infante, M., \& Mora, D. (2012). El papel de los traductores en los tratados de libre comercio (Colombia-Estados Unidos) (trabajo de grado de maestría). Universidad EAN. Recuperado de https://repository.ean.edu.co/bitstream/handle/10882/3024/ InfanteMonica2012.pdf? sequence $=7$ \&isAllowed $=y$

Insignares, J. (2009). ¿Hacia el camino de la profesionalización de la traducción gracias a la futura NTC? Mutatis Mutandis, 2(2), 393-397.

Livio, T. (2000). La traduction jurée au Brésil : Aspects historiques et pratiques. En International colloquium organized by the School of Translation and Interpretation of the University of Geneva and the Swiss Translators', Terminologists' and Interpreters' Association (pp. 1-8). Geneva, Switzerland. Recuperado de http://www.tradulex.com/Actes2000/ cruzromao.pdf

Lobato, J. (2007). Aspectos deontológicos y profesionales de la Traducción jurídica, jurada y judicial (tesis de doctorado). Universidad de Málaga. Recuperado de http://www. biblioteca.uma.es/bbldoc/tesisuma/17114597.pdf

Martín, J. (2013). Traducciones jurídicas: competencias del traductor e impacto en su interpretación (trabajo de grado de maestría). Universidad EAN. Recuperado de https://repository.ean.edu.co/bitstream/handle/10882/5924/MartinJohann2013. pdf? sequence $=4 \&$ isAllowed $=\mathrm{y}$

Martínez, L., \& Guilman, S. (2005). La figura del Traductor Público en la República Argentina. En Coloquio de la Asociación Colombiana de Traductores e Intérpretes (pp. 1-12). Bogotá, Colombia: ACTTI. Recuperado de http://www.cttic.org/ACTI/2004/papers/Ponencia_ Martinez-Guilman.pdf 
Mayoral, R. (1999). Las fidelidades del traductor jurado: Batalla indecisa. En F. García (Ed.), Traducir para la justicia (pp. 17-57). Granada, España.

Mayoral, R. (2000). (Official) Sworn translation and its functions. Babel, 46(4), 300-331. https:// doi.org/10.1075/babel.46.4.03may

Montealegre, I. (2013). Estado actual del mercado de la interpretación en Bogotá (trabajo de grado de maestría). Universidad EAN. Recuperado de https://repository.ean.edu.co/ bitstream/handle/10882/4965/MontealegreIrma2013.pdf? sequence $=1 \&$ isAllowed $=\mathrm{y}$

Monzó, E. (2002). La professió del traductor jurídic $i$ jurat. Descripció sociològica de la professió $i$ anàlisi discursiva del transgènere (tesis de doctorado). Departament de Traducció i Comunicació - Universitat Jaume I. Recuperado de https:/dialnet.unirioja.es/ servlet/tesis?codigo $=6994$

Ordoñez, P. (2009). La evaluación de traductores e intérpretes jurados: Revisión teórica y análisis del tratamiento informativo de los candidatos a pruebas de acreditación y selección. Íkala, Revista de Lenguaje y Cultura, 14(21), 59-81.

Ortega, J. M. (2011). Cómo acreditar intérpretes a través de la traducción: Análisis crítico de la acreditación profesional de intérpretes jurídicos en España. Trans, Revista de Traductología , 15, 131-153. https://doi.org/10.24310/TRANS.2011.v0i15.3207

Ortiz, L., \& Sanabria, A. (2013). Perfil del traductor: incidencia de su formación en el desempeño profesional (trabajo de grado de maestría). Universidad EAN. Recuperado de https://repository.ean.edu.co/bitstream/handle/10882/5065/0rtiz-Sanabria-Tesis. pdf? sequence $=1 \&$ isAllowed $=\mathrm{y}$

Padilla, M. (2018). Analysis of the current status of interpreting as a profession in Colombia: An exploratory case study (trabajo de grado de maestría). Universidad de Pamplona, Colombia.

Pavlović, T. (2013). Exploring Directionality in Translation Studies. Explorations in English Language and Linguistics, 1(2), 149-165.

Peñarroja, J. (1989a). Intérpretes jurados: Documentos para su historia. Boletín de APETI, pp. 1-5.

Peñarroja, J. (1989b). Intérpretes jurados: Documentos para su prehistoria. Boletín de APETI, pp. $1-5$.

Peñarroja, J., \& Cardona, E. (1993). Els intèrprets jurats. Revista de Llengua i Dret, 19, 199-203. 
Perdu, N.-A., \& Ridao, S. (2014). Dificultades de la traducción jurídica y jurada. Tejuelo, 20, 9-26.

Pym, A., Grin, F., Sfreddo, C., \& Chan, A. (2012). The status of the translation profession in the European Union. Luxembourg: Publications Office of the European Union.

Quiroz, G., Gómez, N., \& Zuluaga, J. (2013). Panorama general del traductor e intérprete oficial en Colombia. Núcleo, 30, 165-203.

Quiroz, G., \&Zuluaga,J. (2014). Candidatos a traductor e intérprete oficial en Colombia: Profesionales u oportunistas. Babel, 60(4), 514-538. https://doi.org/10.1075/babel.60.4.06qui

Quiroz, G., Zuluaga, J., \& Gómez, N. (2015). Estatus actual del traductor en Colombia. Medellín, Colombia: Editorial Universidad de Antioquia.

Salvador, S. (1996). El certificat de traducció i interpretació jurades i el registre de traductors i intèrprets jurats: De projecte a realitat. Llengua i ús, 5, 51-54.

Stejskal, J. (2003). International certification study: South America revisited. The ATA Chronicle, 5/2(March), 11-14.

Stejskal, J. (2005). Survey of the FIT Committee for information on the status of the translation \& interpretation profession. FIT: Féderation Internationale de Traducteurs. Recuperado de http://www.uta.org.ua/data/loads/fit_survey_report-2005-07.pdf

Vigier, F. (2010). El nombramiento de traductores-intérpretes jurados de inglés mediante acreditación académica:Descripción de la formación específicaydelgradodesatisfacción de los egresados (tesis de doctorado). Departamento de Traducción e Intepretación Universidad de Granada. Recuperado de https://hera.ugr.es/tesisugr/18896753.pdf

Vigier, F., Klein, N., \& Krestinger, P. (2013). Certified translators in Europe and the Americas: Accreditation practices and challenges. En A. Borja \& F. Prieto (Eds.), New trends in translation studies (pp. 27-51). Oxford, UK: Peter Lang.

Zamora, J. (2005). La traducción oficial en Costa Rica: ¿Una actividad profesional? (Trabajo de grado de maestría). Facultad de Filosofía y Letras - Universidad Nacional de Costa Rica. Recuperado de http://www.acotipcr.org/wp-content/uploads/2015/10/La-traducciin-Oficial-en-Costa-Rica-una-actividad-profesional.pdf

Zuluaga, J. (2014). Certificarse como traductor e intérprete oficial. Alma Mater, p. 15. Recuperado de https://issuu.com/periodicoalmamater/docs/am_634_agosto_de_2014

Zuluaga, J. (2015). Requisito: traducción oficial. Alma mater, p. 30. Recuperado de https://issuu. com/periodicoalmamater/docs/am_634_agosto_de_2014 
Zuluaga, J. (2019). Traducción e interpretación oficial en Colombia. UN Periódico Digital. Recuperado de https://unperiodico.unal.edu.co/pages/blog/detail/traduccion-einterpretacion-oficial-en-colombia/

Zuluaga, J., \& Quiroz, G. (2018). Análisis del desempeño de candidatos a traductor e intérprete oficial en Colombia. Cadernos de Tradução, 38(2), 263-293. https://doi.org/10.5007/21757968.2018v38n2p263 